\title{
Diderot Studies, tome XXXIII, éd. Thierry Belleguic
}

\section{Olga Penke}

\section{OpenEdition}

\section{Journals}

\section{Édition électronique}

URL : http://journals.openedition.org/studifrancesi/1166

DOI : 10.4000/studifrancesi. 1166

ISSN : 2427-5856

\section{Éditeur}

Rosenberg \& Sellier

\section{Édition imprimée}

Date de publication : 1 novembre 2014

Pagination : $597-598$

ISSN : 0039-2944

\section{Référence électronique}

Olga Penke, «Diderot Studies, tome XXXIII, éd. Thierry Belleguic », Studi Francesi [En ligne], 174 (LVIII I

III) | 2014, mis en ligne le 01 novembre 2014, consulté le 18 septembre 2020. URL : http://

journals.openedition.org/studifrancesi/1166; DOI : https://doi.org/10.4000/studifrancesi.1166

Ce document a été généré automatiquement le 18 septembre 2020.

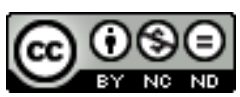

Studi Francesi è distribuita con Licenza Creative Commons Attribuzione - Non commerciale - Non opere derivate 4.0 Internazionale. 


\title{
Diderot Studies, tome XXXIII, éd. Thierry Belleguic
}

\author{
Olga Penke
}

\section{RÉFÉRENCE}

Diderot Studies, tome XXXIII, éd. Thierry BELLEGUIC, Genève, Droz, 2013, pp. XII-349.

1 Cette riche livraison se compose de onze contributions. La majeure partie est consacrée à la présentation de l'état actuel de l'édition critique, entreprise en 1975 et prévue en 33 volumes, dont les derniers paraîtront bientôt grâce aux efforts d'une équipe éditoriale élargie (Les CEuvres complètes de Diderot (DPV): Écrits politiques et correspondance. Les derniers volumes à paraître, pp. 3-266). Suite à la mise au point faite en 2007, la réalisation du premier des objectifs, l'édition du "dernier Diderot" s'est accélérée, tandis que le second, la numérisation du DPV connait des progrès moins spectaculaires (voir sur l'état de ce projet l'article A. Guilbaud et al. dans Recherches sur Diderot et sur l' Encyclopédie, n. 48, 2013, pp. 225-261). Huit études rédigées par les membres de l'équipe éditoriale présentent un dossier précieux où l'on peut trouver les résultats des recherches concernant l'établissement des textes ayant trait à la politique et à la correspondance. Les articles sont suivis d'annexes illustrant le travail en cours. Georges DULAC résume dans l'Avant-propos, écrit en collaboration avec Didier KAHN, les particularités de l'édition des ouvrages de la «période russe» et des lettres (pp. 3-6). Deux études de DULAC, éminent spécialiste de Diderot et la Russie, sont consacrées aux problèmes qui se posent à l'éditeur des Mélanges philosophiques et des Observations sur le Nakaz, adressés à Catherine II (à paraître: DPV 21, 22). La «lecture croisée» des deux discours politiques facilite la reconstitution de l'histoire de leur genèse et l'établissement des principes convenables pour leur édition critique. Dulac exhibe les défauts des éditions antérieures et les préjugés qui ont altéré la lecture des Mélanges philosophiques que Diderot a écrits pendant son séjour en Russie. Il souligne que l'auteur possédait des connaissances étendues et variées sur son sujet, démontre que son intention consistait à la fois à constater l'échec politique, à favoriser la mise en œuvre des solutions 
nouvelles et à offrir à Catherine II un «bon modèle» de société (Les Mélanges philosophiques pour Catherine II: un essai de réhabilitation, pp. 23-82). L'édition des Observations sur le Nakaz, que Diderot compose après son retour, pose un autre type de problème: le choix difficile parmi les copies très différentes. Dulac avoue qu'aucune solution éditoriale ne parait entièrement satisfaisante pour cette œuvre capitale, restée inachevée et probablement jamais destinée à la publication par l'auteur, et expose ses arguments pour préférer comme texte de base la copie la plus ancienne, revue par Diderot. (Les Observations sur le Nakaz: de nouveaux choix éditoriaux, pp. 107-152). Certains textes sur la Russie, présents dans d'autres copies, seront également accessibles pour le lecteur qui les trouvera dans DPV 26, publiant les contributions de Diderot à l'ouvrage de Raynal: l'Histoire des deux Indes qui seront réunies pour la première fois et publiés par les soins éditoriaux de l'éminent spécialiste du langage politique de Diderot, Gianluigi GOGGI. Il résume les recherches scientifiques internationales sur le sujet, auxquelles il ajoute ses résultats remarquables et ses hypothèses bien argumentées (La collaboration de Diderot à l'Histoire des deux Indes: l'édition de ses contributions, pp. 167-212). Pour un autre texte politico-philosophique de Diderot il propose de nouvelles datations, permettant ainsi de révéler l'opposition du philosophe à l'absolutisme, au «despotisme» dès 1763 (Le Discours d'un philosophe à un roi: hypothèses de datation et caractères généraux, pp. 7-22). Deux études mettent sous un jour nouveau les écrits de Diderot au retour de Saint-Pétersbourg, légués à Catherine II. Sergueï KARP décrit le manuscrit et les sources des trois questionnaires de Diderot, adressés parallèlement à Catherine II, à l'Académie des Sciences de Saint-Pétersbourg et à Ernst von Münnich (responsable administratif pour le commerce de la tzarine) et étudie l'origine des informations $\mathrm{du}$ philosophe concernant la vie économique du pays (Les trois questionnaires de Diderot sur la Russie, pp. 83-106). Catherine VOLPILHAC-AUGER établit une savante analyse comparée entre d'une part les Notes écrites par la main d'un souverain à la marge de Tacite, publiées et annotées par Naigeon (1798), des imperfections des différentes éditions jusqu'aujourd'hui, et d'autre part les textes de Tacite accessibles au $\mathrm{xVIII}^{\mathrm{e}}$ siècle, afin de formuler les dangers que l'édition critique en cours d'élaboration devrait éviter: se donner comme but la recherche érudite, répertorier les imprécisions des écrits de Diderot sans connaître les éditions antiques dont il se sert, et se garder de présenter le texte moderne du philosophe par rapport à l'auteur ancien (Les Principes de politique des souverains: le piège de l'érudition, pp. 153-166). L'édition d'un autre ensemble précieux des œuvres de Diderot, sa Correspondance sera également bientôt terminée (DPV 28-32) grâce aux efforts réunis des diderotistes éminents: E. Boussuge, M. LecaTsiomis, G. Cammagre, G. Stenger, sous la responsabilité d'Annie ANGRÉMY et Didier KAHN. Ces deux derniers rendent compte du travail collectif aboutissant aux principes unifiés d'une nouvelle numérotation, qui permettra que toutes les lettres écrites par Diderot et à lui adressées soient répertoriées, y compris celles qui ont été récemment découvertes, celles dont l'athenticité est douteuse, mais aussi celles dont le statut est incertain: il s'agit de celles qui ne sont pas de véritables lettres ou qui ne sont connues que d'allusions (p. ex. à partir des catalogues de vente). Nous y trouvons une liste qui renferme la mise en place des indications - précisions concernant l'original autographe, datation, vérification du texte, etc. - qui peuvent modifier nos connaissances concernant la vie, l'œuvre, la réflexion de Diderot (La nouvelle édition de la Correspondance, pp. 213-263). 
2 Trois études font partie des «Miscellaneous articles». Jean-Pierre CLÉRO analyse l'ambiguïté et l'usage des notions de l'imagination et de la fiction dans la philosophie de Hume et de Bentham, révélant le passage des concepts des forces physiques aux psychiques. Son étude approfondie aboutit à une réflexion sur «l'usage des fictions» par Diderot dans la Religieuse (Le jeu de l'imagination et de la fiction chez Hume et chez Bentham, pp. 267-310). Claire FAUVERGUE repère les articles de l'Encyclopédie dans lesquels on peut reconnaître l'idée de perspective de Leibniz, sa méthode de l'analogie ou bien le rapport établi entre l'esprit, l'âme et le corps de sa Monadologie (Le leibizianisme et l'histoire de la philosophie dans l'Encyclopédie, pp. 311-324). Laurence MARIE démontre les relations intimes entre la réflexion de Diderot sur le comédien - qui maîtrise les symptômes du sentiment et parvient au refus de l'imitation de la nature au profit de celle du modèle idéal - et sur le poète, le peintre, l'artiste ainsi que sur leur rapport avec le public, dans ses œuvres sur l'art de la fin des années 1750 au début des années 1770 (Diderot et la relation du comédien à son rôle: du Fils naturel au Paradoxe sur le comédien, pp. 325-349).

3 Ce volume de Diderot Studies témoigne du relancement intense des activités scientifiques et éditoriales en 2013, anniversaire du tricentenaire de la naissance du philosophe, qui peuvent contribuer à la meilleure compréhension de l'œuvre de Diderot. 